of the systematies and distribution of all animals already found, or expected to occur, in the Soviet territories. Six volumes and two smaller parts, mostly dealing with insects, have already appeared, and they make a very good impression, particularly as regards the generally high scientific standard, although the paper, printing and illustrations might be improved. Unfortunately, not all the volumes are by outstanding experts in the systematics of the groups treated, and some have apparently been prepared by authors who may be excellent specialists, but not in the groups they had to monograph. All foreign zoologists will be pleased to see that each volume has a very full summary in a Western language, in which all determination keys, new descriptions and more important notes are given. Since the "Fauna". covers the whole of extra-tropical Asia and eastern Europe, its value for systematists working on the palæarctic region will be inestimable, and volumes on their respective groups will be eagerly awaited by all zoologists.

\section{The Botanical Society of Edinburgh}

The Transactions and Proceedings of the Botanical Society of Edinburgh, 32, Part 1, 1936, is a particularly interesting number, including as it does the record of the centenary meeting of this Society. The main contribution is in effect a local flora-a list of the flowering plants and ferns from Fife and Kinross by William Young. The address delivered by Prof. F. O. Bower at the centenary meeting is included. No more appropriate speaker could have been found for that interesting occasion, and his appreciation of botanical progress during the period of activity of the Society could only have been delivered by a veteran whose reminiscences still feed his botanical enthusiasm. Prof. Bower points out how plant physiology has gained since 1836 by the precision that can now be assigned to the medium in which vital functions are proceeding, through the advances in cytology and anatomy. With arresting phrase and breadth of vision, in a few pages the main movements of botanical thought during the century are brought before the reader. The last half century, it is pointed out, because it is an age of specialization, has increased the need for the services of such general societies as the Botanical Society of Edinburgh. Prof. Bower indicates how the 'herd sense' among his fellow botanists is probably responsible for the temporary ascendancy of one field of investigation, thus leading to "multiplying instances of what has been already demonstrated". He then shows himself fully aware where the 'herd' is gathering now when he ends an eloquent plea for a modern morphology, based upon developmental studies of the meristem, with the speculation that hormones may then prove the key to unlock those phenomena of symmetry that are expressed in appendages. The Botanical Society of Edinburgh began principally as a means to the formation of a herbarium for its members, and has taken a prominent part in the creation of the magnificent herbarium that is now housed at the Royal Botanic Gardens.

\section{Giorgi's System of Units}

Dr. A. E. Kenneliy recently presented a report, from the historical point of view, to the Society for the Promotion of Engineering Education, in regard to the adoption of the M.K.S. (metre-kilogram-second) system of units ("The M.K.S. System of Giorgi as adopted by the International Electrotechnical Commission (I.E.C.) in June 1935"). Dr. Kennelly has done his work well, giving a fair statement of the present position. Unfortunately, physicists and engineers are not yet agreed as to the best system of units to adopt. Many of them are in favour of following Heaviside and completely rationalizing the system of units. Others are more conservative, and think that the change, although saving much arithmetic, would scarcely be worth the labour involved in learning all the relations connecting the new and the old units. The two leading organizations are in agreement that a fourth unit is theoretically necessary to link internationally adopted electrical units with the dynamical units of the M.K.s. system. They agree that this fourth unit may preferably be 'space permeability' taken at the definite numerical value of $10^{-7}$ (unrationalized). This report of Dr. Kennelly's can do nothing but good.

\section{Prohibition and Cirrhosis of the Liver}

AT an address given at the recent annual meeting of the Royal Institute of Public Health at Margate, Dr. C. C. Weeks stated that, in the United States, when prohibition really was prohibiting, there was a marked decrease in the death-rate from cirrhosis of the liver and a rise as 'boot-legging' became more extensive. The liquor sold under 'boot-legging' conditions was much more alcoholic than usual, ethyl alcohol being so high as 70 per cent in much of the whisky sold. There was consequently a good deal of acute alcoholism attributed to all sorts of causes, whereas the one effective cause was that the whisky was 50 per cent stronger than usual. Since the repeal of prohibition, there has been a slight but steady increase in deaths due to alcoholism. Official figures for 1935 showed that, out of 24 States, 20 had an increase in the death-rate from cirrhosis, while only 11 showed an increase in the death-rate for alcoholism as compared with 1932. The steady drinking of a more normal alcoholic beverage was leading inevitably to more cirrhosis of the liver but to less acute alcoholism.

\section{Society of Chemical Industry: Food Group}

AT a recent meeting of the Food Group of the Society of Chemical Industry, it was decided, in view of the growing interest in food science and the diversity of the subject, to form panels within the Group. These panels will be responsible for directing the activities of the Group in connexion with their respective divisions of the science. The first two panels, of which there will be several, are now in process of formation, namely, a nutritional panel and a microbiological panel. 